\title{
APPLICATION OF A CONTINUOUS WAVELET TRANSFORMS AND ANFIS TO FAULT DIAGNOSIS OF A GEARBOX OF TRACTOR
}

\author{
Mostafa Bahrami ${ }^{1}$, Hossein Javadikia ${ }^{1 *}$, Ebrahim Ebrahimi ${ }^{2}$ \\ ${ }^{1}$ Department of Mechanical Engineering of Agricultural Machinery, Faculty of Agriculture, \\ Razi University, Kermanshah, Iran \\ ${ }^{2}$ Department of Mechanical Engineering, Faculty of Engineering, \\ Kermanshah Branch Islamic Azad University, Kermanshah, Iran \\ *Corresponding e-mail: pjavadikia@gmail.com
}

\begin{abstract}
This study proposes a technique based on continuous wavelet transform added by adaptive-neural-fuzzy inference system (ANFIS) for fault diagnosis of gearbox of MF285 tractor. Varying fault can cause changes in a measured gearbox vibration signal. A gear motion vibration signal in speed of $1500 \mathrm{r} / \mathrm{min}$ is analyzed as source data. Energy of coefficients wavelet is proposed to evaluate tractor gear fault by ANFIS. We found that this technique can correctly indicate early gear fault.
\end{abstract}

Keywords: MF285 tractor; gearbox; fault detection; continuous wavelet transform

\section{INTRODUCTION}

Condition monitoring and crack identification in dynamic structures are key areas of engineering research. Scientist are focusing on their attention and analyzing various methodologies for fault identification and condition monitoring ${ }^{1}$ and need to attention to tractor gearbox fault by new technique too.

Different approaches for fault detection and diagnosis successful proposed. Most of these techniques involve vibration analysis because they are easy to measure and highly reliable ${ }^{2}$. Some of the recent efforts made by engineers for fault identification on tractor are proposed, such as Fault diagnosis of Massey Ferguson gearbox using power spectral density ${ }^{3}$. Intelligent fault classification of a tractor starter motor using vibration monitoring and adaptive neural-fuzzy inference system ${ }^{4}$. Developing an Intelligent Fault Diagnosis of MF285 Tractor Gearbox Using Genetic Algorithm and Vibration Signals ${ }^{5}$.Classifier fusion of vibration and acoustic signals for fault diagnosis and classification of planetary gears are based on Dempster-Shafer evidence theory ${ }^{6}$ and Some of the recent efforts made by engineers for fault identification on other application are proposed, such as induction motors bearing fault detection using pattern recognition techniques ${ }^{7}$. Exploiting sound signals for fault diagnosis of bearings using decision tree $^{8}$. Fault diagnosis of induction motor based on decision trees and adaptive neural-fuzzy inference ${ }^{2}$. Feature extraction using wavelets and classification through decision tree algorithm for fault diagnosis of mono-block centrifugal pump ${ }^{9}$.

The aim of this study provides a procedure based on a continuous wavelet transform added by adaptive-neural- fuzzy inference system for fault diagnosis of gearbox of MF285 tractor.

\section{MATERIALS AND METHOD \\ Data acquisition}

To study the number's one gear of gear box of MF285 tractor failure, our experiment does with one speed (1500 $\mathrm{r} / \mathrm{min}$ ) and three conditions (normal condition, worn gear and broken gear). In this paper, the signal was got by an accelerometer mounted on the bearing of experimental set up, when it was loaded in the speeds.

The experimental setup to collect dataset also consists of analyzer system, an accelerometer, tachometer and shock absorbers under the base of test-bed, as shown in Fig. 1. Experimental setup was designed to install number's one gear of gear box of MF285 tractor, electric motor and load mechanism with shock absorbers under bases to cancel out vibrations. Iteration rate for each case is 130 and 4 second time. The rotational speed of the system was measured by tachometer.

\section{Continuous wavelet transform and feature extraction}

The continuous wavelet transform is the sum over all time of the signal multiplied by scaled, shifted versions of the wavelet. This process produces wavelet coefficients that are a function of scale and position.

It's really a very simple process. In fact, there are the five steps of an easy recipe for creating a CWT:

1. Take a wavelet and compare it to a section at the start of the original signal.

2. Calculate a number, $\mathrm{C}$, that represents how closely correlated the wavelet is with this section of the signal. The higher $\mathrm{C}$ is, the more the similarity. More precisely, if the signal energy and the wavelet energy

Journal of Mechanical Engineering, Vol. ME 46, December 2016

Transaction of the Mechanical Engineering Division, The Institution of Engineers, Bangladesh 
are equal to one, $\mathrm{C}$ may be interpreted as a correlation coefficient (see Fig. 2).

Note that the results will depend on the shape of the wavelet is chosen. In this paper DB4 family is chosen.

3. Shift the wavelet to the right and repeat steps 1 and 2 until cover the whole signal.

4. Scale (stretch) the wavelet and repeat steps 1 through 3.

5. Repeat steps 1 through 4 for all scales.

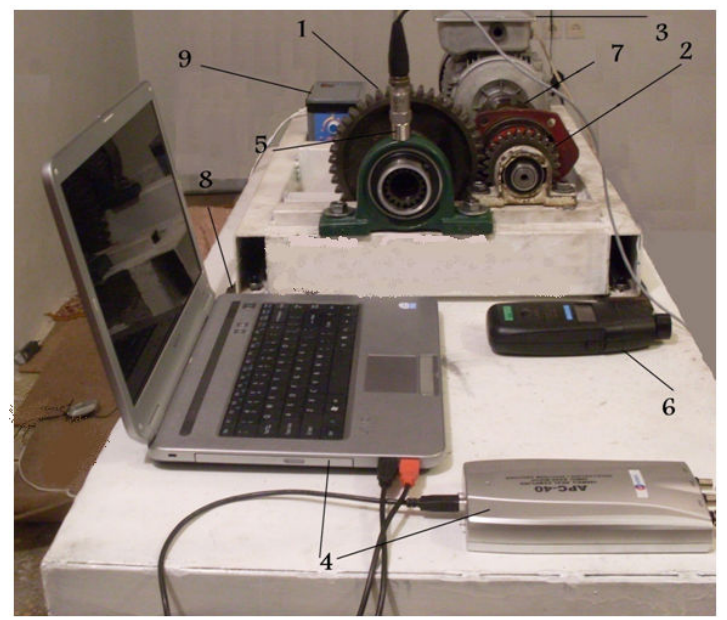

Figure 1. Experimental setup.

1 - gear, 2 - small gears, 3 - Electric motor , 4 - data captures and laptop, 5 - Accelerometer sensor, 6 - optical tachometer, 7 - coupling, 8 - Vibration absorbing and 9 - Industrial dimmer.

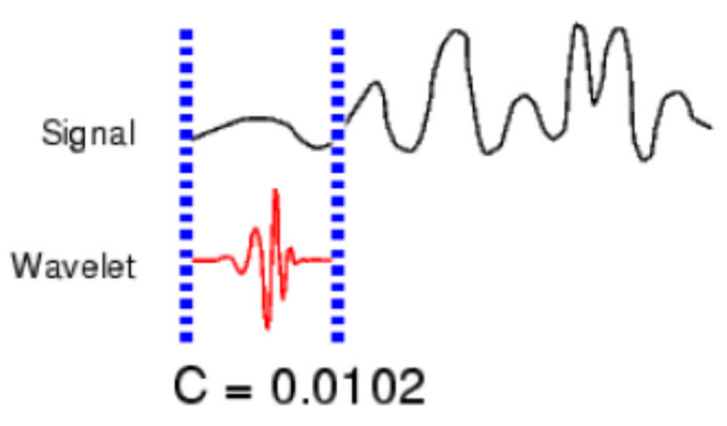

Figure 2. Continuous Wavelet Transform

When steps are done, the coefficients produced at different scales by different sections of the signal. The coefficients constitute the results of a regression of the original signal performed on the wavelets [10].For sense of these entire coefficients surface on which the $x$-axis represents scale, the $y$-axis represents position along the signal ( 250 data equal to 720 degree spin of gear), and the color at each $Z$ point represents the magnitude of the wavelet coefficient $\mathrm{C}$. These are the coefficient plots generated by the graphical tools (Figure 3, Figure 4 and Figure 5).
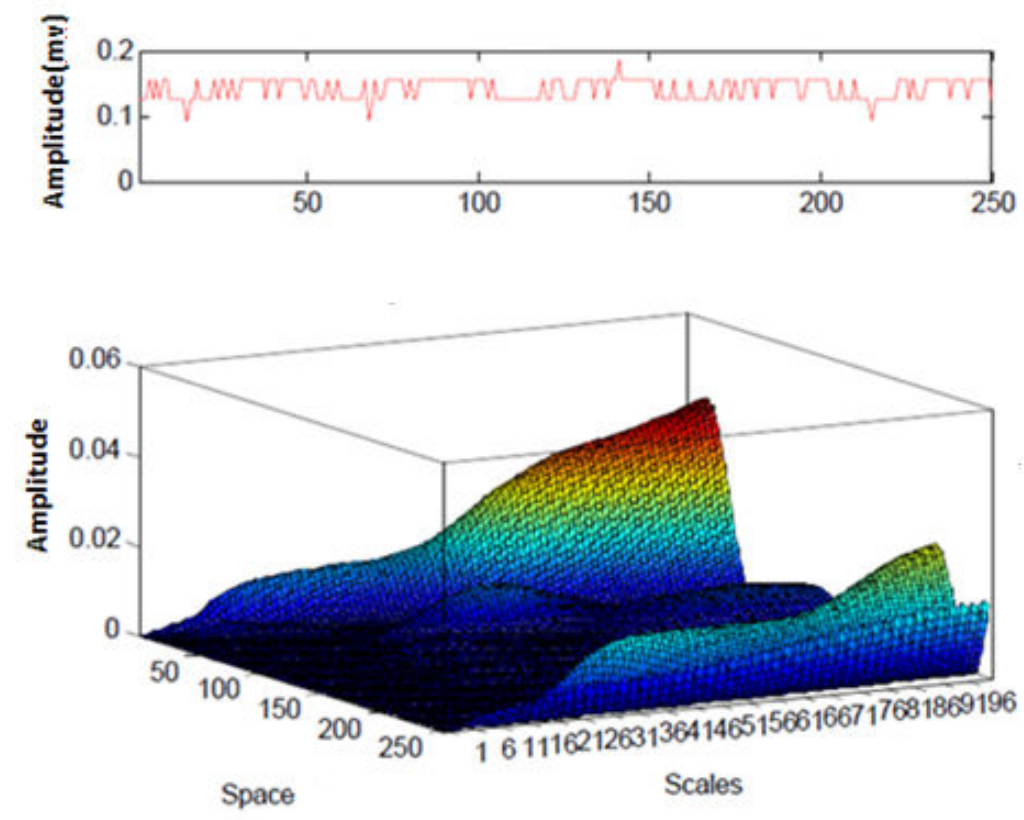

Figure 3. Signal related to normal condition (top), entire coefficients surface (down)

Journal of Mechanical Engineering, Vol. ME 46, December 2016

Transaction of the Mechanical Engineering Division, The Institution of Engineers, Bangladesh 

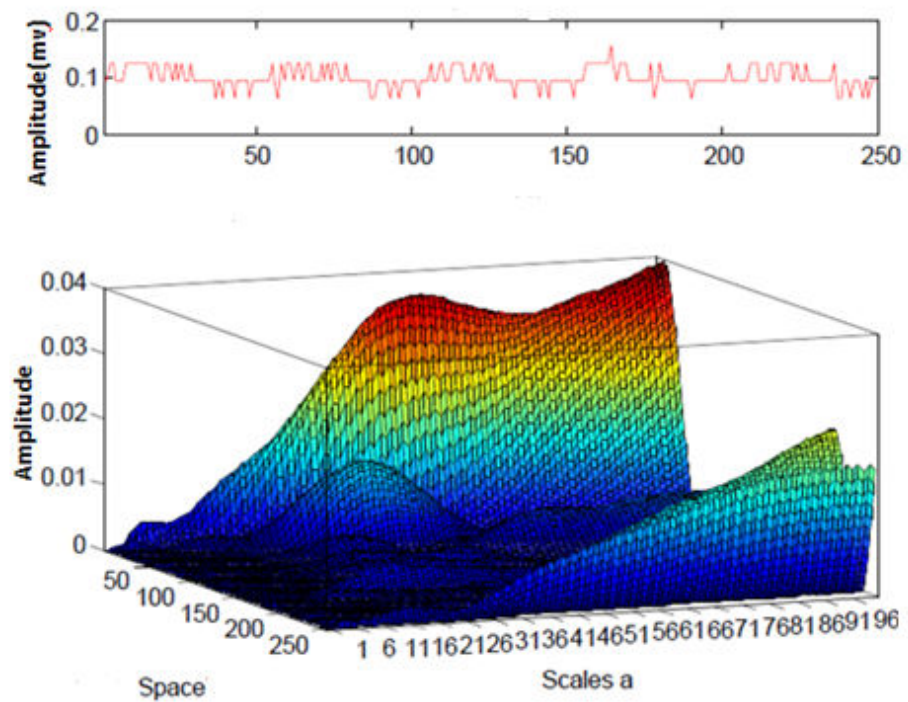

Figure 4. Signal related to gear worn condition (top), entire coefficients surface (down).
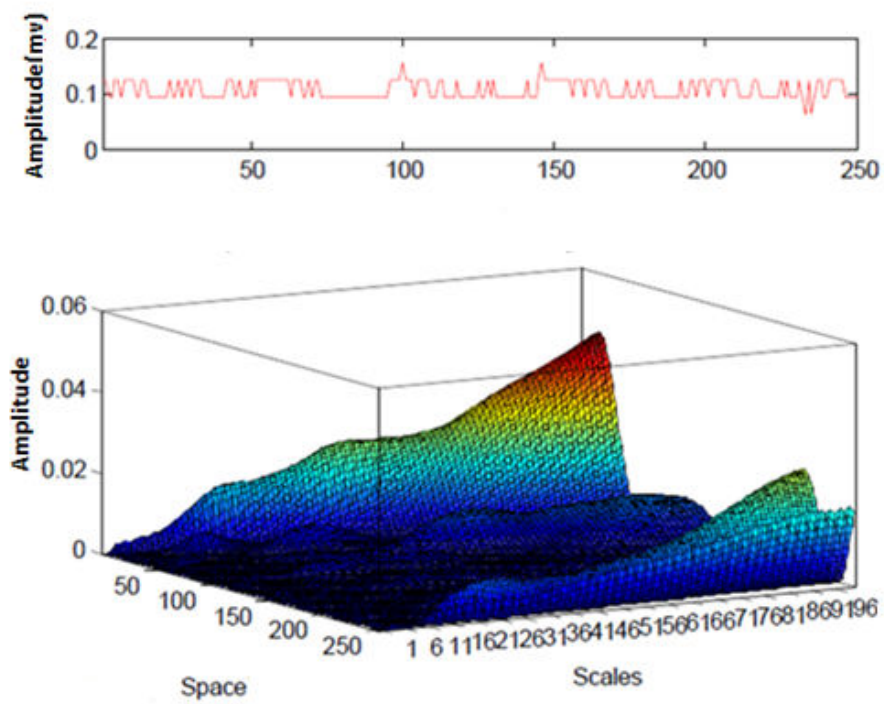

Figure 5. Signal related to gear broken condition (top), entire coefficients surface (down).

For feature extraction, first sum of wavelet coefficients energy of 100 scale of 250 data point (equal to 720 degree spin of gear) are calculated and one row of 250 data is achieved. Then two features (Standard deviation and Skewness) of 250data are calculated. These two features are given in following Equation 1 and Equation 2.

$$
\text { Standard Deviation }=\sqrt{\frac{\sum_{n-1}^{N}\left(\left(x(n)-\frac{\sum_{n-1}^{N} x(n)}{N}\right)^{2}\right.}{N-1}}
$$

Journal of Mechanical Engineering, Vol. ME 46, December 2016

Transaction of the Mechanical Engineering Division. The Institution of Engineers, Bangladesh 
Skewness $=$

$$
\frac{n}{(n-1)(n-2)} \sum_{n-1}^{N}\left(\frac{x(n)-\frac{\sum_{n-1}^{N} x(n)}{N}}{\sqrt{\frac{n \sum_{n-1}^{N} x^{2}(n)-\sum_{n-1}^{N}(x(n))^{2}}{n(n-1)}}}\right)^{3}
$$

where $x(n)$ is a scales series for $n=1,2, \ldots, N$, $\mathrm{N}$ is number of data points( 250 data points).

\section{ANFIS}

As reported by ${ }^{11,12}$ was the first person that proposed an adaptive neural-fuzzy inference system (ANFIS). An adaptive neural-fuzzy inference system (ANFIS) structure is composed of five layers. (I) the fuzzification layer, (II) the rule layer,(III) the normalization layer, (IV) the defuzzification layer and (V) the output layer and the output layer. In this study, modeling was performed using ANFIS Toolbox in MATLAB (R2009b).The fuzzy structures, which were used in the fault classification, are based on Sugeno fuzzy model based on a set of input-output data. Fuzzy models are widely investigated. Proposed model is shown in Figure 6. In this study 390 sample, normal(130sample), gear worn (130sample) and gear broken(130sample),sap were tested by the system, and were divided into two sets, 70\% for training set and 30\% for testing set.

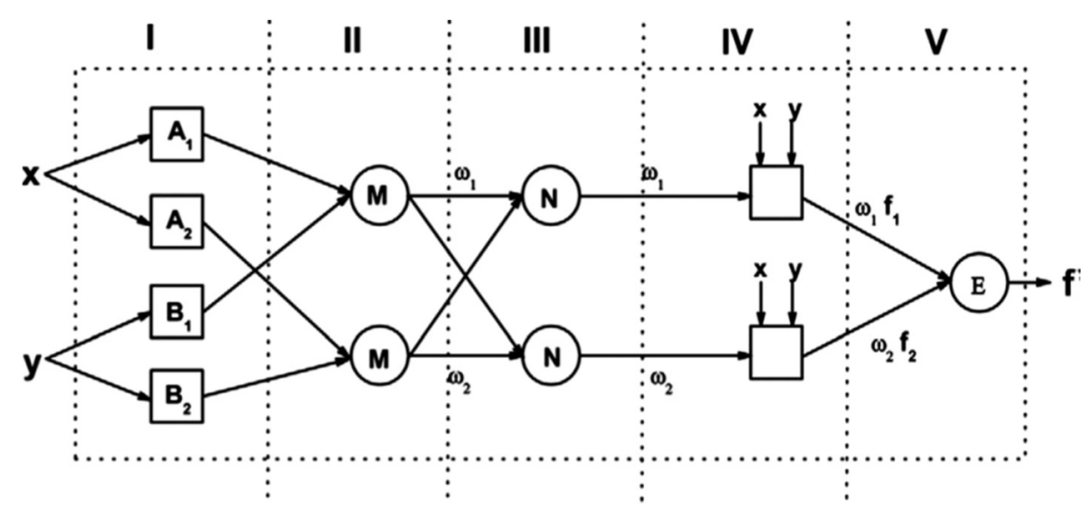

Figure 6. ANFIS structure (Sabzy et al.,2013)

\section{EXPERIMENTAL RESULTS}

The aim of this paper is to design an adaptive neuralfuzzy inference system model using continuous wavelet transforms features. For this purpose, the two features consist of Standard deviation and Skewness of the Continuous Wavelet Transform coefficients, are used as the inputs of the ANFIS. So, the two input vectors for the ANFIS is applied. The architecture of a sample inference system is indicated in Fig. 7.

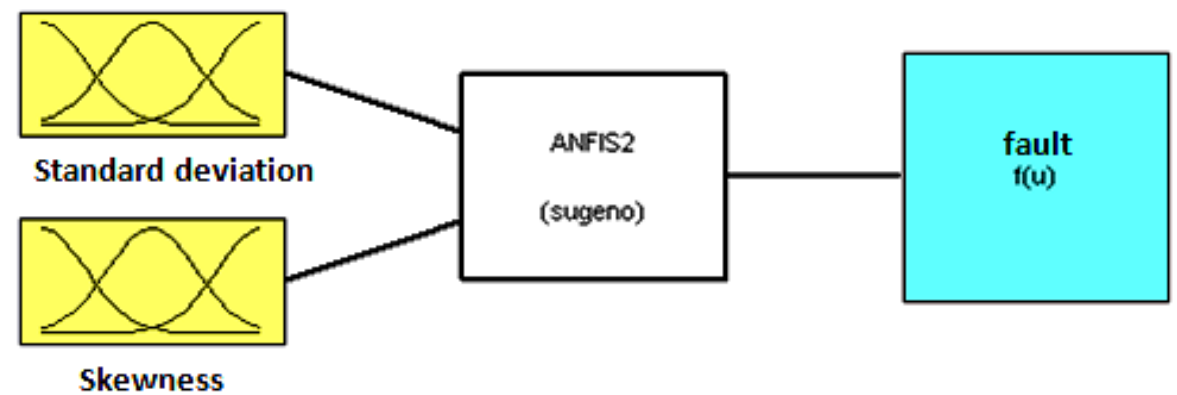

Figure 7. The architecture of inference system

Journal of Mechanical Engineering, Vol. ME 46, December 2016

Transaction of the Mechanical Engineering Division, The Institution of Engineers, Bangladesh 
The dataset divided in two part; training ( $70 \%$ dataset) and test (30\% dataset).

The best structure of the ANFIS model was obtained based on the minimum value of the error of the training and testing set. Number of membership function for inputs, constant and linear type membership function that are utilized for output during generating IS, optimum methods and iterations were investigated and the results were indicated in Table 1. As can be seen, the minimum error of testing sets obtained values of 0.000903. ANFIS structure of best model is shown in
Figure 8.Therefore, the ANFIS model with the linearity of output MFs type, 2, 6 numbers of Gaussmf for input, hybrid of optimum method was selected as the best model. Figure 9 show error versus the number of iterations for the best ANFIS model. As can be seen, the training error goes steadily after about 6 epochs. The predicted values of data in the testing sets using the ANFIS model and their plotting versus experimental data were displayed in Figure10. For the testing data set, the coefficient of determination $\left(\mathrm{R}^{2}\right)$ is 0.9988 .

Table 1. Comparison of different ANFIS model

\begin{tabular}{ccccccc}
\hline Model number & Input $\mathrm{mf}$ structure optimum method & Input $\mathrm{mf}$ & Out-put $\mathrm{mf}$ & $\mathrm{MSE}$ & $\mathrm{R}^{2}$ \\
\hline 1 & {$[26]$} & hybrid & linear & Gaussmf & 0.000903 & 0.9988 \\
2 & {$[4]$} & hybrid & linear & Gaussmf 2 & 0.0022 & 0.9973 \\
3 & {$[26]$} & hybrid & linear & Pimf & 0.0320 & 0.9570 \\
4 & {$[34]$} & hybrid & constant & Dsigmf & 93.4 & 0.6759 \\
5 & {$[25]$} & hybrid & constant & Tri mf & 0.3959 & 0.4745 \\
\hline
\end{tabular}

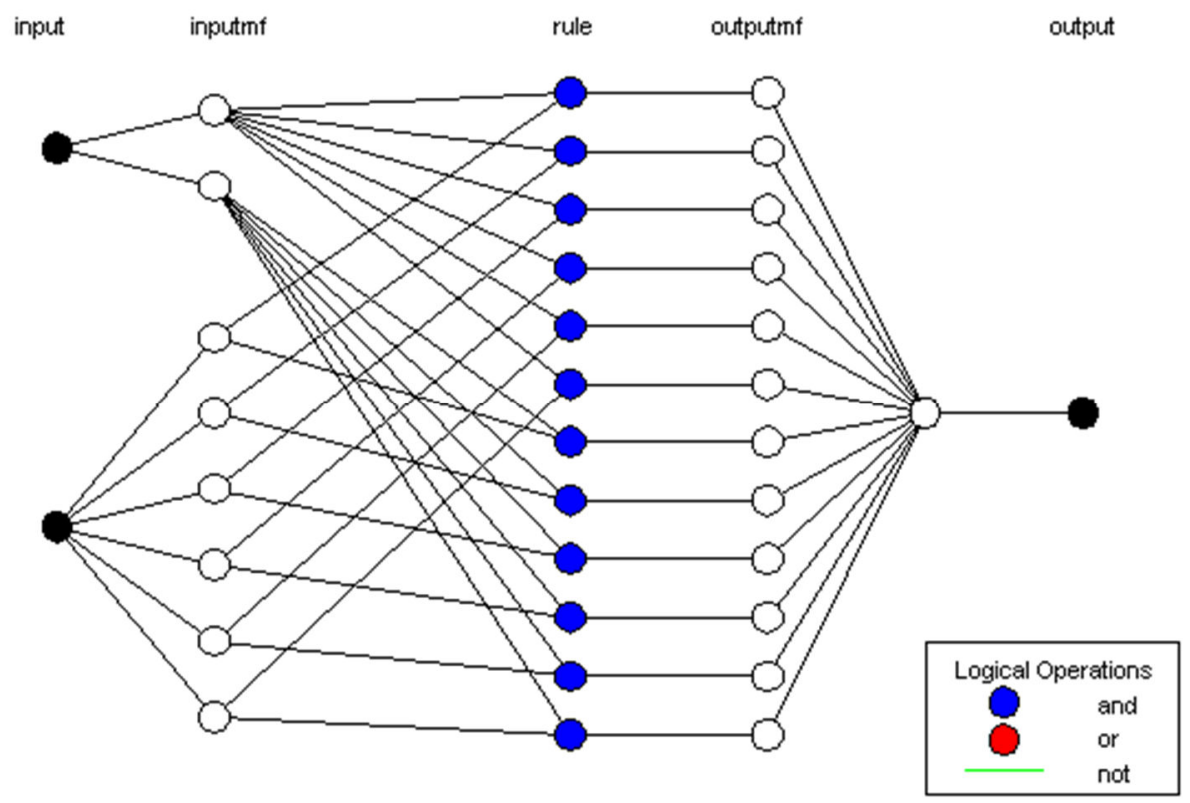

Figure 8. ANFIS structure of best model

Journal of Mechanical Engineering, Vol. ME 46, December 2016 


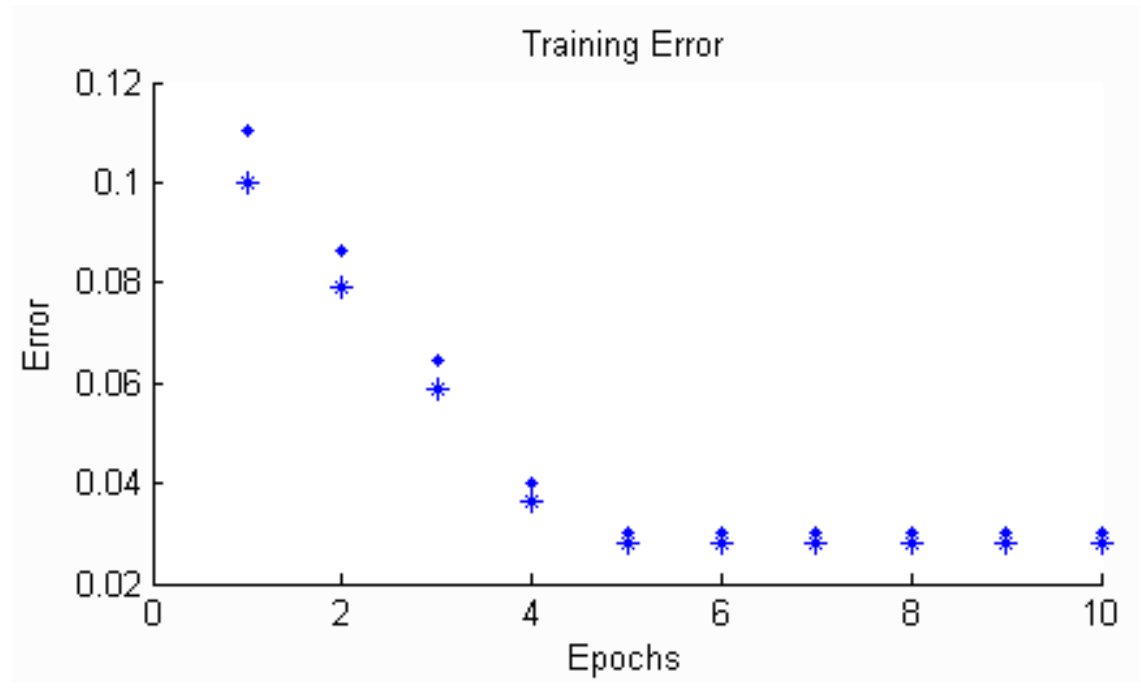

Figure 9. Error versus the number of epochs.

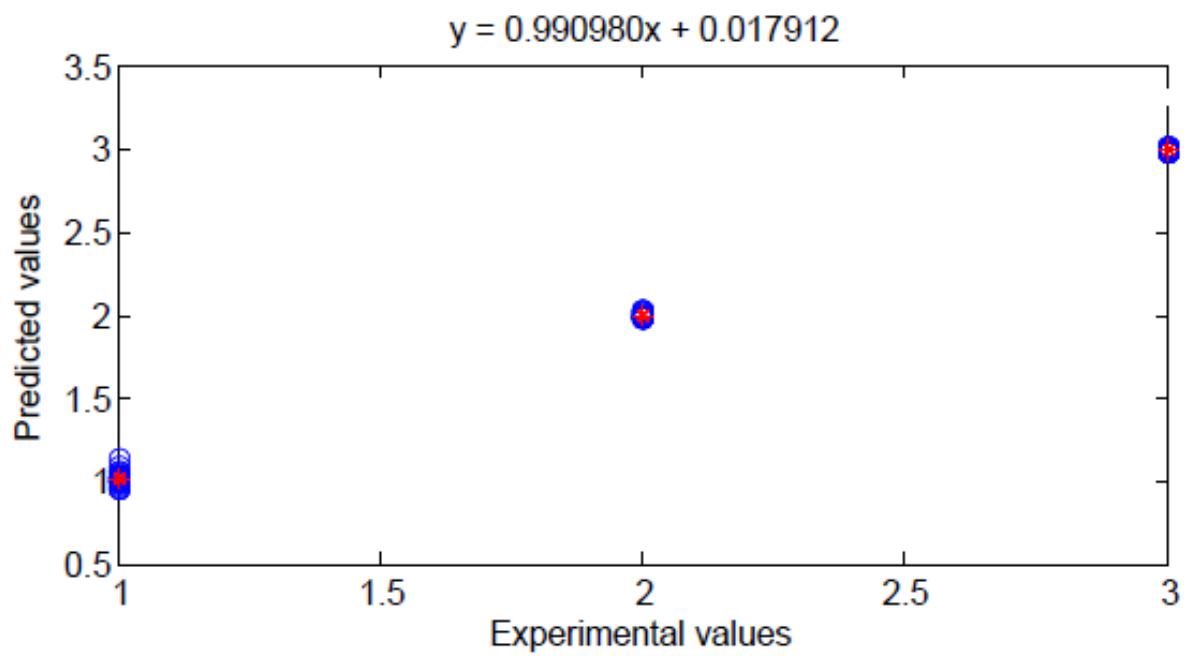

Figure 10. The comparison of experimental and ANFIS predicted values.

\section{CONCLUSIONS}

The aim of this study is use of continuous wavelet transform added by adaptive-neural-fuzzy inference system (ANFIS) to intelligent fault diagnosis of gearbox of MF285 tractor. In first, an experimental set-up was designed, and the proper data was acquired in three conditions, such as normal condition, gear worn and broken worn. Then data is processed by continuous wavelet transform and two features (Standard Deviation and Skewness) by statistical equation are got. Different adaptive-neural-fuzzy inference system model were designed. In first, 70\% data is applied as the inputs of the ANFIS for training set and then the other $30 \%$ data uses as the testing of the ANFIS model. It is shown that using these features have acceptable accurate for fault diagnosis.

\section{Acknowledgment}

Special thanks to Mr. Mohamad Hadi Jalili and Mr. Nasrolah Astan for helping this research.

\section{REFERENCES}

1. Parhi, D.R.K. and Das, H., 2010. Diagnosis of fault and condition monitoring of dynamic structures using the multiple adaptive-neuro-fuzzy inference system technique. Proceedings of the Institution of 
Mechanical Engineers, Part G: Journal of Aerospace Engineering, 224(3), pp.259-270.

2. Tran, V.T., Yang, B.S., Oh, M.S. and Tan, A.C.C., 2009. Fault diagnosis of induction motor based on decision trees and adaptive neuro-fuzzy inference. Expert Systems with Applications, 36(2), pp.1840-1849.

3. Heidarbeigi, K., Ahmadi, H. and Omid, M., 2008, September. Fault diagnosis of Massey Ferguson gearbox using power spectral density. In Electrical Machines, 2008. ICEM 2008. 18th International Conference on (pp. 1-4). IEEE.

4 Ebrahimi,E., and Mollazade.K. 2010. Intelligent fault classification of a tractor starter motor using vibration monitoring and adaptive neuro-fuzzy inference system. Insight - Non-Destructive Testing and Condition Monitoring, 52 (10): 561-566.

5. Ebrahimi, E., Javadikia, P., Astan, N., Heydari, M., Bavandpour, M., Jalili, M.H. and Zarei, A., 2013. Developing an Intelligent Fault Diagnosis of MF285 Tractor Gearbox Using Genetic Algorithm and Vibration Signals. Modern Mechanical Engineering, 3(04), p.152.

6. Khazaee, M., Ahmadi, H., Omid, M., Moosavian, A. and Khazaee, M., 2014. Classifier fusion of vibration and acoustic signals for fault diagnosis and classification of planetary gears based on DempsterShafer evidence theory.Proceedings of the Institution of Mechanical Engineers, Part E: Journal of Process Mechanical Engineering, 228(1), pp.21-32.
7. Zarei, J., 2012. Induction motors bearing fault detection using pattern recognition techniques. Expert systems with Applications, 39(1), pp.68-73.

8. Amarnath, M., Sugumaran, V. and Kumar, H., 2013. Exploiting sound signals for fault diagnosis of bearings using decision tree. Measurement, 46(3), pp.1250-1256.

9. Muralidharan, V. and Sugumaran, V., 2013. Feature extraction using wavelets and classification through decision tree algorithm for fault diagnosis of monoblock centrifugal pump. Measurement, 46(1), pp.353359.

10. Von Gizycki, H., 2008. Long Distance Functional Coupling Associated with EEG State Changes: The Examination of Phase and Amplitude Synchrony of Neural Assemblies Across Hemispheres During REM and Non-REM Sleep Onset. ProQuest.

11. Jang, J.S., 1993. ANFIS: adaptive-network-based fuzzy inference system.IEEE transactions on systems, man, and cybernetics, 23(3), pp.665-685.

12. Sabzi, S., Javadikia, P., Rabani, H. and Adelkhani, A., 2013. Mass modeling of Bam orange with ANFIS and SPSS methods for using in machine vision. Measurement, 46(9), pp.3333-3341.

Journal of Mechanical Engineering, Vol. ME 46, December 2016 